\title{
Telemonitoring in chronic ventilatory failure: a new model of survellaince, a pilot study
}

\author{
S. Bertini, M. Picariello, M. Gorini, T. Renda, A. Augustynen, G. Villella, \\ G. Misuri, N.M. Maluccio, R. Ginanni, D. Tozzi, A. Corrado
}

ABSTRACT: Telemonitoring in chronic ventilatory failure: a new model of survellaince, a pilot study. S. Bertini, M. Picariello, M. Gorini, T. Renda, A. Augustynen, G. Villella, G. Misuri, N.M. Maluccio, R. Ginanni, D. Tozzi, A. Corrado.

Background and Aim. The efficiency of tele-monitoring or tele-assistance in patients with severe chronic ventilatory failure in home mechanical ventilation (HMV) is still being investigated. Our aim was to test the feasibility of a model which consisted in: 1) once a week nocturnal telemonitoring, supervised by a doctor in charge in a Respiratory Intensive Care Unit, who also provided a telephone-counselling (24/7) on demand; 2) a scheduled visit every two months.

Methods. A 2-year observational study was carried out on 16 patients ventilated for at least 1 year and for $\geq 8$ hours/day. Once a week patients underwent a nocturnal monitoring during HMV. The compliance was evaluated by regular transmission of data and regular follow-up, the level of satisfaction by a telephonequestionnaire.

Results. The adherence to the protocol study was good in 9/16 $(56 \%)$ and poor in $7 / 16(44 \%)$ patients. For each patient, the mean number of connections was $46,12 \pm 36.39$ ( $70.7 \%$ of that expected), in those with good compliance it increased to $63.8 \pm 32.7$ (114\% of that expected). The median hours of connection was 343 (138$1019)$ and $89(0-521)$ for patients with good and poor compliance respectively, $\mathrm{p}=\mathbf{0 . 0 3 8}$. The mean scheduled visits for patient with good compliance was $6.9 \pm 4.14$ (100\% of that expected). Emergency visits were avoided in $62.5 \%$ of cases. The satisfaction score was higher in compliant versus non compliant patients $(p=0.019)$.

Conclusion. This pilot study showed that the telemonitoring system employed was feasible and effective in more compliant patients who claimed a high rate of satisfaction.

Monaldi Arch Chest Dis 2012; 77: 2, 57-66.

Keywords: Chronic Respiratory Failure, Home Mechanical Ventilation, Respiratory Intensive Care Unit, Telehealth, Telemedicine.

Respiratory Intensive Care Unit, AOU - Careggi, Firenze, Italy.

Correspondence: Dr. Stefania Bertini, Respiratory Intensive Care Unit and Thoracic Physiopathology Laboratory, AOU Careggi,Florence,Italy; e-mail: bertinis@aou-careggi.toscana.it

\section{Introduction}

The number of patients with chronic respiratory failure (CRF) who need ventilatory assistance at home is increasing. This is due mainly to two factors: an aging population which is associated to an increase of chronic respiratory disorders [1] and patients who survived an acute respiratory disease but need to be supported with continuous mechanical ventilation at home [2]. These patients have a severe prognosis and are subjected to frequent hospitalisation which in turn determines a worsening of the quality of life and an increase of healthcare costs [3-5].

The role of telemedicine is emerging as support of care for patients with advanced disability [6-10], it may represent a useful tool for home care for frail patients with respiratory disorders [10]. The American Thoracic Society statement on home care for patients with respiratory disorders [11] recommends a strict follow-up of these patients which in turn may translate in a reduction of complications resulting from hospitalisation, maintaining an acceptable quality of life; and en- abling a comfortable and dignified death in terminally ill patients [11]. The follow-up of patients in home mechanical ventilation should be integrated with technologies $[2,12]$. These should permit remote control of the ventilator and modify in real time its setting, according to the patient's conditions, by a clinical operator [13].

A recent metanalysis [14] has reported that in Chronic Obstructive Pulmonary Disease (COPD) patients home telehealth (home tele-monitoring and telephone support), compared with usual care, reduced rates of hospitalisation and emergency department visits. Home telehealth intervention were similar or better than usual care for quality of life and patients satisfaction. This data has been confirmed recently [15], however the benefit of telemonitoring for COPD is not yet proven and further work is required before wide scale implementation be supported [16] Different models of telemonitoring [17-19] and tele-assistance [10, 20, 14] have been reported.

The aim of the present study was to evaluate in patients with advanced chronic ventilatory failure in home mechanical ventilation a new model of 
telemonitoring, in order to verify its feasibility and its impact on the perception of the patients in term of the service, safety, and facilitation in the disease management. Furthermore the impact of this model on the course of the disease in terms of hospital admission and length of stay were also considered.

This telemonitoring model consisted in: 1) a once a week nocturnal monitoring and a telephone counselling, when needed, available 24 hour/24, 7 days per week with medical doctors in charge in a Respiratory Intensive Care Unit (RICU); 2) regular visits every 2 months.

\section{Methods}

This pilot observational study lasting two years was carried out from 1 st October 2008 to 30th September 2010 at the outpatient clinic division of Respiratory Intensive Care Unit of Azienda Ospedaliera Careggi, Firenze.

The inclusion criteria were: chronic ventilatory failure (CVF), home mechanical ventilation (HMV) for at least 1 year, 8 or more hours of mechanical ventilation per day, domiciliary in Florence or in its province.

Exclusion Criteria were: obstructive sleep apnoea (OSA), no telephone access at home, no caregivers to facilitate telephone contact, residence in a nursing home, illiteracy, and refusal. The patients who underwent a clinical control at our outpatient clinic division from the 1 st January to the $30^{\text {th }}$ June 2008 and who fulfilled the inclusion/exclusion criteria were screened and considered for inclusion in the study.

All patients included in the study and their caregivers were well accustomed in the use of the ventilator which had already been assigned to them for at least one year. The training was performed by a respiratory physician before the commencement of home mechanical ventilation. All patients received at home a digital multiparametric recorder (SALLY PA, QBGROUP Spa - Padova, Italy). The system was installed at home by a technician who, in the presence of a doctor involved in the study, instructed the patient/caregivers on the use of the system. As reported by Tura [21] the SALLY recorder allows acquisition and storage of multiple non-invasive clinical data (Tidal Volume, Respiratory Frequency, Minute Ventilation, Arterial Oxygen Saturation $\left(\mathrm{SaO}_{2}\right)$, Heart rate, Inspiratory Positive Airway pressure (IPAP), Expiratory Positive Airway Pressure (EPAP)). The data acquired was stored and transmitted, on demand, via Internet at a station located in RICU. Once a week, all patients underwent overnight home monitoring during mechanical ventilation with registration of the above variables. Furthermore a telephone counselling of 24/24 hours and 7 days/week with the doctors in charge in RICU was provided when needed. Acute medical problems and alarms generated by the ventilator were immediately reported by phone to the doctor in charge in RICU, who, in real time, looking at the monitorised traces, was able to suggest the solution for the specific problem. If the problem was impossible to resolve from a distance the doc- tor suggested calling an ambulance for transfer of the patient to the emergency department. If the patient's condition proved very serious, hospitalisation in RICU was recommended.

Patients were checked with regular visits every two months at the outpatient clinic of RICU.

The compliance to the study protocol was evaluated as good (regular transmission of data and regular follow-up), or poor (no regular or absent transmission of data and regular, no regular or absent follow-up).

At each visit, the patients were evaluated regarding: clinical history with particular emphasis on the stability status and pharmacological treatment; clinical examination and arterial blood gas analysis; the level of dyspnoea evaluated by the MRC scale [22]; occurrence of hospitalisation (number and duration); occurrence of urgent General Practitioner (GP) call and relative cause; occurrence of unscheduled visits at the outpatient clinic of RICU and relative cause; control of ventilator setting and relative accessories; and compliance to the ventilatory treatment and number of hours/day of use of ventilator.

Spirometry was performed at the start of the study, when possible, using a spirometer $\operatorname{Vmax}_{22}$ (Sensor Medics, Yorba Linda, CA, USA), in according to ATS/ERS standard procedure [23].

The comorbidities were registered for each patient. The evaluation of the impact of the tele-monitoring on the perception of the patients in terms of usefulness of the service, safety, and facilitation in the management of the disease was assessed, at the end of the study, through a telephone questionnaire. The questionnaire consisted of 3 items with 4 answers for each item and a score for each item ranging from 0 to 3 , with 0 as negative response, 1 as little, 2 as moderate and 3 as complete level of satisfaction. The total scoring number ranged from 9 (maximum level of satisfaction) and 0 (minimum level of satisfaction). The level of satisfaction was expressed as percentage of the maximum score ranging from 0 to $100 \%$. A score higher than $5 / 9$ $(56 \%)$ was considered the lowest limit of acceptable satisfaction. In appendix $\mathrm{A}$ is reported the telephone questionnaire. The questionnaire was administered to the patients or to the care givers.

The general practitioner (GP) was involved in the study for an integrated management of the patient.

Each patient was used as control of his/her self in order to compare the number of hospitalisation and the days of hospital stay registered during the study period versus two years before the commencement of the study.

All patients gave written informed consent after the protocol approval by the local ethics committee

\section{Statistical Analysis}

Results are expressed as mean \pm SD for normally distributed variables, and median (range), with $95 \%$ Interval of Confidence for no normally distributed variables. The non parametric method for paired (Wilcoxon) and unpaired (Mann With- 
ney) data was used to compare non normally distributed variables; Chi square test and Student's t test were used when appropriate. Differences were considered statistically significant at $p<0.05$.

\section{Results}

\section{Characteristics of patients}

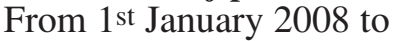
30th June 2008, 72 patients with CVF in HMV in followup at our outpatient clinic were screened. In fig. 1 is reported the trial profile of the enrolled patients. Among the 23 patients that met the inclusion criteria, 7 died before the installation of the device at home. The chronic disease underlying CVF of the 7 patients who died were: Amyotrophic Lateral Sclerosis (ALS) in 4 patients ( 3 in invasive and 1 in noninvasive HMV); COPD in 2 patients (both in Long Term Oxygen Therapy and noninvasive HMV), Muscular Dystrophy (MD) in 1 patient treated at home with noninvasive HMV.
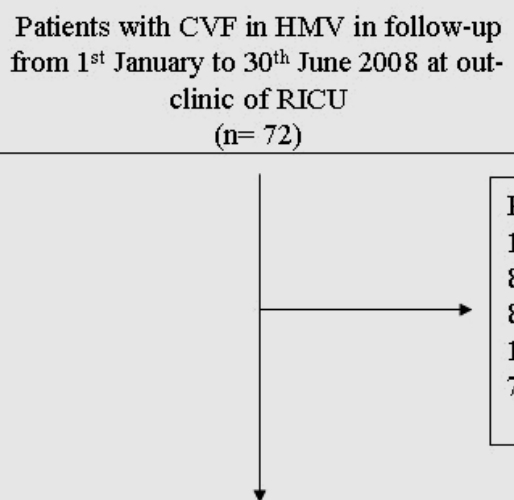

Patients with exclusion criteria $\mathrm{n}=49$ $16=$ lived out of province $8=$ refused

$8=$ OHS

$10=$ OSA

$7=$ unfavourable environmental conditions

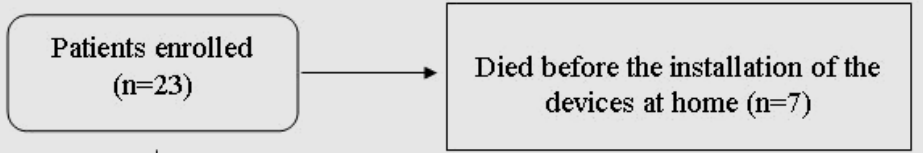

Patients studied $(\mathrm{n} .=16)$

Fig. 1. - Trial profile of the enrolled patients.

In table 1 are reported the baseline characteristics of patients at the start of the study. In table 2 are reported the type of disease, type of mechanical ventilation, hours/die of treatment and comorbidities. Five patients ( 2 with ALS, 2 with

Table 1. - Antropometric, clinical carattericts, arterial blood gases, $\mathrm{pH}$, oxygen therapy and ventilator setting of patients with chronic ventilatory failure in home mechanical ventilation at the start of the study

\begin{tabular}{|c|c|c|c|c|c|c|c|}
\hline & No pats & Mean & SD & Median & $95 \% \mathrm{CI}$ & Min & Max \\
\hline Age, years & 16 & 59 & 17 & 59 & $50,28-71,36$ & 20 & 85 \\
\hline Height (m) & 16 & 1,63 & 0,132 & 1,66 & $1,49-1,73$ & 1,44 & 1,80 \\
\hline BMI & 16 & 26 & 6 & 25 & $21,14-29,24$ & 15 & 39 \\
\hline MRC scale & 16 & 4 & 1,6 & 4 & $3,00-5,00$ & 0 & 5 \\
\hline BP max & 16 & 123 & 16 & 123 & $110-134$ & 100 & 150 \\
\hline BP min & 16 & 73 & 9 & 70 & $70-80$ & 60 & 90 \\
\hline $\mathrm{RR}(\mathrm{cpm})$ & 16 & 22 & 5 & 20 & $20-24$ & 16 & 38 \\
\hline HR (bpm) & 16 & 87 & 15 & 92 & $75-100$ & 64 & 104 \\
\hline $\mathrm{FEV}_{1} \%$ pred & 11 & 52 & 32 & 41 & $25-77$ & 17 & 123 \\
\hline VC \% pred & 11 & 55 & 30 & 53 & $29-78$ & 16 & 111 \\
\hline MIP \% pred & 7 & 38 & 13 & 41 & $21-51$ & 20 & 54 \\
\hline MEP \% pred & 7 & 29 & 10 & 32 & $18-39$ & 15 & 43 \\
\hline $\mathrm{PaO}_{2} \mathrm{mmHg} \S$ & 16 & 82 & 15 & 80 & $72-91$ & 64 & 110 \\
\hline $\mathrm{PaCO}_{2} \mathrm{mmHg} \S$ & 16 & 47 & 11 & 47 & $43-52$ & 26 & 69 \\
\hline $\mathrm{pH} \S$ & 16 & 7,40 & 0,05 & 7,39 & $7,37-7,43$ & 7,33 & 7,52 \\
\hline $\mathrm{HCO}_{3} \mathrm{mmMol} \S$ & 16 & 28 & 5 & 27 & $24-32$ & 22 & 37 \\
\hline $\mathrm{SaO}_{2} \%$ & 16 & 96 & 2 & 96 & $95-98$ & 92 & 99 \\
\hline Hrs of MV/day ** & 16 & 14 & 7,3 & 10 & $8-23$ & 8 & 24 \\
\hline Months of MV ** & 16 & 74 & 74 & 54 & $33,5-72,0$ & 20 & 312 \\
\hline $\mathrm{O}_{2}$ in $\mathrm{SB}(\mathrm{L} / \mathrm{min})$ & 16 & 0,38 & 0,59 & 0,00 & $0,00-0,93$ & 0,0 & 1,5 \\
\hline $\mathrm{O}_{2}$ in $\mathrm{MV}(\mathrm{L})$ & 16 & 0,73 & 1,014 & 0,00 & $0,00-1,18$ & 0,0 & 3,0 \\
\hline EPAP $\mathrm{cmH}_{2} \mathrm{O}$ & 16 & 4 & 1 & 4 & $4-4$ & 2 & 6 \\
\hline IPAP $\mathrm{cmH}_{2} \mathrm{O}$ & 16 & 16 & 3 & 16 & $14-17$ & 8 & 20 \\
\hline
\end{tabular}

BMI = Body mass index; MRC $=$ Medical Research Council Scale; $\mathrm{BP}=$ Blood Pressure; RR = Respiratory Rate; HR $=$ Heart Rate; $\mathrm{FEV}_{1}=$ Forced Expiratory Volume in $1 \mathrm{sec}$; $\mathrm{VC}=$ Vital capacity; MIP = Maximal Inspiratory Pressure; MEP = Maximal Expiratory Pressure; MV = mechanical ventilation; $\mathrm{SB}=$ Spontaneous Breathing; EPAP = Expiratory Positive Airway Pressure; IPAP = Inspiratory Positive Airway Pressure; §in five patients in home mechanical ventilation 24/24 hrs/day: $\mathrm{PaO}_{2}=88.2+17.6 ; \mathrm{PaCO}_{2}=38.2+9.0 ; \mathrm{pH}=7.44+0.06 ; \mathrm{HCO}_{3}=26.4+6.3(\mathrm{Mean}+\mathrm{SD}) ; * *$ pre study. 
Table 2. - Type of disease, mechanical ventilation, hours/day of treatment and comorbidities

\begin{tabular}{|c|c|c|c|c|c|c|}
\hline Patient & Type of Disease & IMV* & IMV hrs/die & NIV§ & NIV hrs/day & Comorbidities \\
\hline OD & OS & & & 1 & $8 / 24$ & Bradycardia, Peace maker \\
\hline $\mathrm{CR}$ & COPD & & & 1 & $8 / 24$ & $\begin{array}{l}\text { Arterial Hypertension, Diabetes, } \\
\text { Atrial fibrillation, Colon Cancer }\end{array}$ \\
\hline $\mathrm{HM}$ & M.D. & 1 & $24 / 24$ & & & Dilated Cardiomyopathy \\
\hline $\mathrm{BM}$ & C.W.D & & & 1 & $8 / 24$ & Arterial Hypertension, Osteoporosis \\
\hline MF & M.D. & 1 & $24 / 24$ & & & Arrhythmia \\
\hline $\mathrm{BB}$ & C.W.D. & & & 1 & $8 / 24$ & Hypothyroidism, Obesity, Hypertension \\
\hline GF & ALS & & & 1 & $16 / 24$ & $\begin{array}{l}\text { Arterial Hypertension, Ischemic cardiopathy, } \\
\text { Chronic renal failure }\end{array}$ \\
\hline $\mathrm{AR}$ & ALS & 1 & $24 / 24$ & & & Diabetes \\
\hline MG & COPD & 1 & $24 / 24$ & & & Arterial hypertension \\
\hline $\mathrm{CaF}$ & M.D. & & & 1 & $12 / 24$ & None \\
\hline $\mathrm{CF}$ & ALS & 1 & $24 / 24$ & & & Chronic renal failure \\
\hline FL & M.D. & & & 1 & $13 / 24$ & Arterial Hypertension, Dilated cardiomyopathy \\
\hline SN & C.W.D. & & & 1 & $8 / 24$ & Arterial Hyperrtension \\
\hline MR & COPD & & & 1 & $8 / 24$ & Lung cancer \\
\hline FV & C.W.D. & & & 1 & $8 / 24$ & Chronic renal failure \\
\hline FR & M.D. & & & 1 & $8 / 24$ & Kidney stones, dolicocolon \\
\hline
\end{tabular}

*IMV = Invasive Mechanical Ventilation; § NIV = Noninvasive Mechanical Ventilation.

OS: Ondine Sindrome, COPD: Chronic Obstructive Pulmonary Disease, M.D.: Muscular Dystrophy, C.W.D.: Chest Wall Deformities, ALS: Amyotrophic Lateral Sclerosis.

MD, and 1 with COPD) were tracheotomised and ventilated $24 / 24$ hours, whereas 11 patients were ventilated with mask ventilation for 8 or more hours/day.

\section{Compliance to study protocol}

The adherence to the protocol study resulted good in 9/16 (56\%) patients and poor in $7 / 16$ (44\%). Two patients dropped out at 10 and 14 months respectively: one who transferred to another region and one for personal reasons (he/she never accepted the idea to be monitored during the night at home). In table 3 the number of connections for each patient, scheduled visits and hours of connection expected on the basis of months of follow-up and those actually effected are reported. In all 16 patients the percentages of effective connections and scheduled visits in comparison to those expected were $70.7 \%$ and $70.5 \%$ respectively. The percentage increased up to $114.9 \%$ and $100 \%$ in patients with good compliance. Moreover the mean number of effective hours of connection was $78.3 \%$ of that expected in all patients and $126.7 \%$ in patients with good compliance.
In table 4 the total hours of connection, number of hours for each connection, number of traces recorded as a whole and for each connection in all sixteen patients and in those with good and poor compliance are reported. The connection for each session lasted for each patient with good compliance a median of 7.13 hours (range 6.19-10.4) and 6.6 (range $0-10.2$ ) in those with poor compliance $(p=0.425)$.

The number of traces transmitted varied according to the type of ventilatory treatment: 7 traces for patients treated with noninvasive mechanical ventilation (NIV) and 11 for those with invasive mechanical ventilation (IMV). In all patients the total number of traces transmitted for each session was a median of 7 (range 0-11); in patients with good compliance 7 (range 6-11) and in patients with poor compliance 4 (range $0-8)(p=$ $0.025)$.

In fig. 2 the mean $\mathrm{SaO}_{2}$ values of the 9 patients with good compliance during the months of follow-up are shown. In fig. 3 and 4 are reported examples of the recorded traces with the Sally System. 


\begin{tabular}{|c|c|c|c|c|c|c|c|c|c|c|c|}
\hline Patient & $\begin{array}{l}\text { Months } \\
\text { of } \\
\text { follow-up }\end{array}$ & $\begin{array}{l}\text { Number of } \\
\text { connections } \\
\text { expected }\end{array}$ & $\begin{array}{l}\text { Effective } \\
\text { connections }\end{array}$ & $\%$ & $\begin{array}{l}\text { Expected } \\
\text { visits }\end{array}$ & $\begin{array}{l}\text { Real } \\
\text { visits }\end{array}$ & $\%$ & Compliance & $\begin{array}{c}\text { Hours of } \\
\text { connection } \\
\text { expected }\end{array}$ & $\begin{array}{l}\text { Effective } \\
\text { hours of } \\
\text { connection }\end{array}$ & $\%$ \\
\hline OD & 24 & 96 & 96 & 100 & 12 & 12 & 100 & GOOD & 672 & 684 & 101,79 \\
\hline $\mathrm{CR}^{\circ}$ & 4 & 16 & 17 & 106 & 1 & 1 & 100 & GOOD & 112 & 138 & 123,21 \\
\hline HM & 24 & 96 & 98 & 102 & 12 & 12 & 100 & GOOD & 672 & 1019 & 151,64 \\
\hline $\mathrm{BM}$ & 24 & 96 & 113 & 118 & 12 & 12 & 100 & GOOD & 672 & 801 & 119,2 \\
\hline MF* & 10 & 40 & 40 & 100 & 5 & 5 & 100 & GOOD & 280 & 264 & 94,286 \\
\hline $\mathrm{BB}^{\circ}$ & 5 & 20 & 39 & 195 & 3 & 3 & 100 & GOOD & 140 & 323 & 230,71 \\
\hline $\mathrm{GF}^{\circ}$ & 12 & 48 & 48 & 100 & 6 & 6 & 100 & GOOD & 336 & 297 & 88,393 \\
\hline $\mathrm{AR}^{\circ}$ & 10 & 40 & 49 & 123 & 5 & 5 & 100 & GOOD & 280 & 343 & 122,5 \\
\hline $\mathrm{MG}^{\circ}$ & 12 & 48 & 74 & 154 & 6 & 6 & 100 & GOOD & 336 & 567 & 168,75 \\
\hline $\begin{array}{l}\text { Mean good } \\
\quad( \pm \mathrm{SD})\end{array}$ & & $\begin{array}{l}55.5 \\
32.3\end{array}$ & $\begin{array}{l}63.8 \\
32.7\end{array}$ & 114.9 & $\begin{array}{l}6.9 \\
4.13\end{array}$ & $\begin{array}{c}6.9 \\
4.13\end{array}$ & 100 & & $\begin{array}{l}389 \\
226\end{array}$ & $\begin{array}{l}493 \\
292\end{array}$ & 126.7 \\
\hline $\mathrm{CaF}$ & 24 & 96 & 20 & 21 & 12 & 12 & 100 & POOR & 672 & 188 & 27,976 \\
\hline $\mathrm{CF}$ & 24 & 96 & 43 & 45 & 12 & 10 & 83 & POOR & 672 & 439 & 65,327 \\
\hline FL & 24 & 96 & 11 & 12 & 12 & 2 & 17 & POOR & 672 & 56 & 8,3333 \\
\hline SN & 24 & 96 & 79 & 82 & 12 & 0 & 0 & POOR & 672 & 521 & 77,53 \\
\hline $\mathrm{MR}^{\circ}$ & 4 & 16 & 11 & 69 & 2 & 0 & 0 & POOR & 112 & 89 & 79,464 \\
\hline $\mathrm{FV}^{\wedge}$ & 14 & 48 & 0 & 0 & 7 & 5 & 71 & POOR & 336 & 0 & 0 \\
\hline FR & 24 & 96 & 0 & 0 & 12 & 0 & 0 & POOR & 672 & 0 & 0 \\
\hline $\begin{array}{l}\text { Mean poor } \\
\quad( \pm S D)\end{array}$ & & $\begin{array}{l}77.7 \\
32.6\end{array}$ & $\begin{array}{l}23.4 \\
28.5\end{array}$ & 30.1 & $\begin{array}{l}9.57 \\
3.82\end{array}$ & $\begin{array}{l}4.12 \\
5.04\end{array}$ & 43.0 & & $\begin{array}{l}544 \\
228\end{array}$ & $\begin{array}{l}185 \\
213\end{array}$ & 34.0 \\
\hline $\begin{array}{l}\text { Mean total } \\
( \pm \mathrm{SD})\end{array}$ & & $\begin{array}{l}65.25 \\
33.29\end{array}$ & $\begin{array}{l}46.12 \\
36.39\end{array}$ & 70.7 & $\begin{array}{l}8.06 \\
4.10\end{array}$ & $\begin{array}{l}5.68 \\
4.16\end{array}$ & 70.5 & & $\begin{array}{l}457 \\
233\end{array}$ & $\begin{array}{l}358 \\
297\end{array}$ & 78.3 \\
\hline
\end{tabular}

${ }^{\circ}$ died; * drop-out at 10 months from the start of the study for transferring in another region, during the 10 monthsof follow-up the patient showed a good compliance; $\wedge$ the patient dropped out deliberately for personal reasons (he/she never accepted the idea to be monitored during the night at home)

\section{Health interventions required during the study and adopted measures}

During the period of the study we received 8 urgent calls from patients: in 5 cases, the doctor in charge in RICU, after the analysis of traces which did not show pathological alterations, reassured the patients and suggested an appropriate medical treatment for the control of symptoms. In this way hospitalisation was avoided in all the 5 cases $(62.5 \%)$. On the contrary in the other 3 cases, the doctor suggested, on the basis of symptoms reported and of the analysis of the oxygen saturation traces, the admission to the Emergency Room. Of these patients: 2 were transferred in RICU ( 1 for Pneumothorax and 1 for pneumonia), and 1 was transferred in pneumology ward for exacerbation of COPD.

During the scheduled visits 2 patients were admitted directly to RICU for increasing dyspnoea and deterioration of arterial blood gases due to an increase of bronchial secretions.
In one patient, after the analysis of traces, the control visit was anticipated. The patient was maintained at home with a modification of the setting of ventilator and a prescription of an appropriate pharmacological treatment. Furthermore a close monitoring for three consecutive nights was prescribed. The prompt and appropriate intervention resulted in a clinical improvement of the patient who was treated safely at home without hospitalisation.

In one patient the analysis of traces suggested a modification of ventilator setting which resulted in a clinical improvement.

During the study, the patients underwent 12 visits by the GP and 3 unscheduled visits at the outpatient clinic division for a total of 15 interventions. Patients with no adherence to the study protocol presented a mean of $1,17 \pm 2,04$ interventions versus $0,8 \pm 1,03$ for those adherent $(p=0.712)$. In eleven cases the call to the GP was 
Table 4. - Total hours of connection, number of hours for each connection, number of traces recorded as a whole and for each connection in all sixteen patients and in those with good and poor compliance

\begin{tabular}{lcccc}
\hline & $\begin{array}{c}\text { All patients } \\
\text { Median } \\
\text { (range) }\end{array}$ & $\begin{array}{c}\text { Good Compliace } \\
\text { Median } \\
\text { (range) }\end{array}$ & $\begin{array}{c}\text { Poor Compliance } \\
\text { Median } \\
\text { (range) }\end{array}$ & $\begin{array}{c}\text { P } \\
\text { for comparison between } \\
\text { good and poor compliance }\end{array}$ \\
\hline No Pts & 16 & 9 & 7 & $* 0.038$ \\
\hline Total No h. connection & $310,0(0,0-1019)$ & $343(138-1019)^{*}$ & $89(0,0-521)^{*}$ & ${ }^{\circ} 0.425$ \\
\hline No h. for each connection & $7,11(0,0-10,40)$ & $7,13(6,19-10,4)^{\circ}$ & $6,6(0,0-10,2)^{\circ}$ & $\wedge 0.009$ \\
\hline Total No of traces recorded & $316(0-814)$ & $539(119-814)^{\wedge}$ & $46(0,0-344)^{\wedge}$ & $\S 0.025$ \\
\hline No of traces for each connection & $7(0-11)$ & $7(6-11) \S$ & $4(0,0-8) \S$ & \multirow{2}{*}{. }
\end{tabular}

The comparison of median between patients with good and poor compliance was carried out by means of Mann-Withney Runk Sum Test

made for the presence of signs of respiratory infection which required a short course of antibiotic treatment, in one case for the presence of arrhythmia and acute hypotension, and in 3 cases for fever.

\section{Hospitalisation and Hospital Stay}

In all patients, the mean number of hospitalisations due to acute respiratory failure in the two years before the study was $0.8125 \pm 0.98$ which decreased to $0.4375 \pm 1.09$ at the end of the study (46\% of reduction); in patients with good compliance it decreased from $1.222 \pm 1.09$ to $0.333 \pm 0.5$ ( $73 \%$ of reduction); whereas in patients with poor compliance it increased from $0.285 \pm 0.487$ to $0.571 \pm 1.133$ (100\% of increase).

The mean days spent in hospital in the two years before the commencement of the study were $9.8125 \pm 16.07$ which decreased to $4.375 \pm 7.9$ at

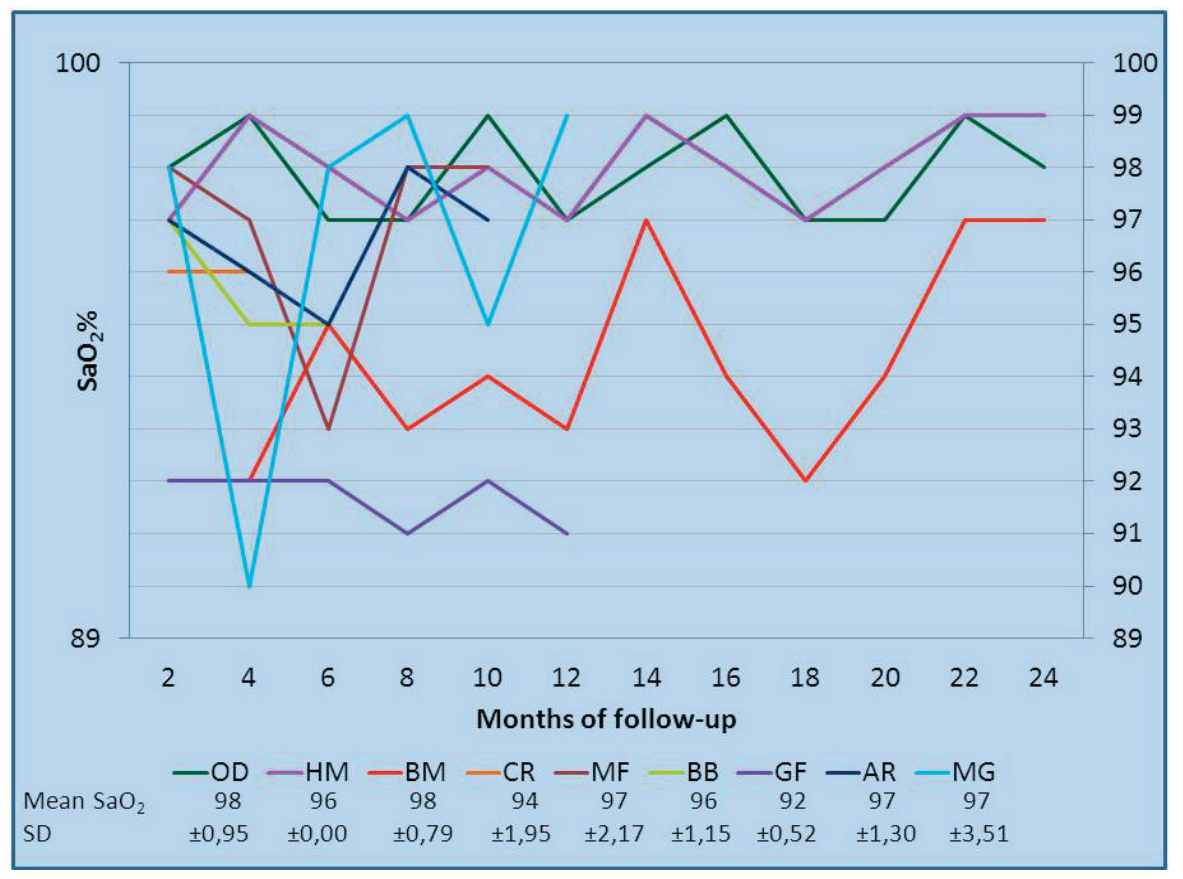

Fig. 2 - Mean $\mathrm{SaO}_{2}$ values of the 9 patients with good compliance during the months of follow-up. the end of the study ( $55 \%$ of reduction); in patients with good compliance it decreased from $16.0 \pm$ 19.4 to $2.6 \pm 4.2$ (83\% of reduction); and in patients with poor compliance it increased from $1.9 \pm$ 3.8 to $6.6 \pm 11.2$ (253\% of increase). A statistical significant difference was ascertained both for the number of hospital admission $(p=0.021)$ and the hospital stays $(p=0.048)$ favouring the group with good adherence to the protocol (table 5).

\section{Outcome}

Of the 16 enrolled patients, 2 died after 4 months from start of study (1 from colon cancer, 1 from lung cancer), 1 after 5 months from severe pneumonia, 2 after 10 and 12 months (both for worsening of underlying disease), 1 after 12 months from Pneumonia and Pneumothorax. Among the 6 patients who died, all in LTOT lasting for more than 5 years, 3 were afflicted with COPD, 2 with ALS and 1 with Chest Wall Deformity. The median survival at 24 months was $62.5 \%$.

Satisfaction score by telephone questionnaire

The satisfaction index in survivors was $67 \%$. When we look at, in a separate way, the patients adherent to the protocol $v s$. those non adherent we found a statistically significant increase in score points $(8 / 9$, median 8,0 , interquartile range 6,0 to $9,0)$ in the first group vs. (3/9, median 3,5 , interquartile range 1,0 to $5,0)$ in the second $p=$ 0.019 . The satisfaction index was $89 \%$ in the adherent vs. $33 \%$ in the non adherent group. 


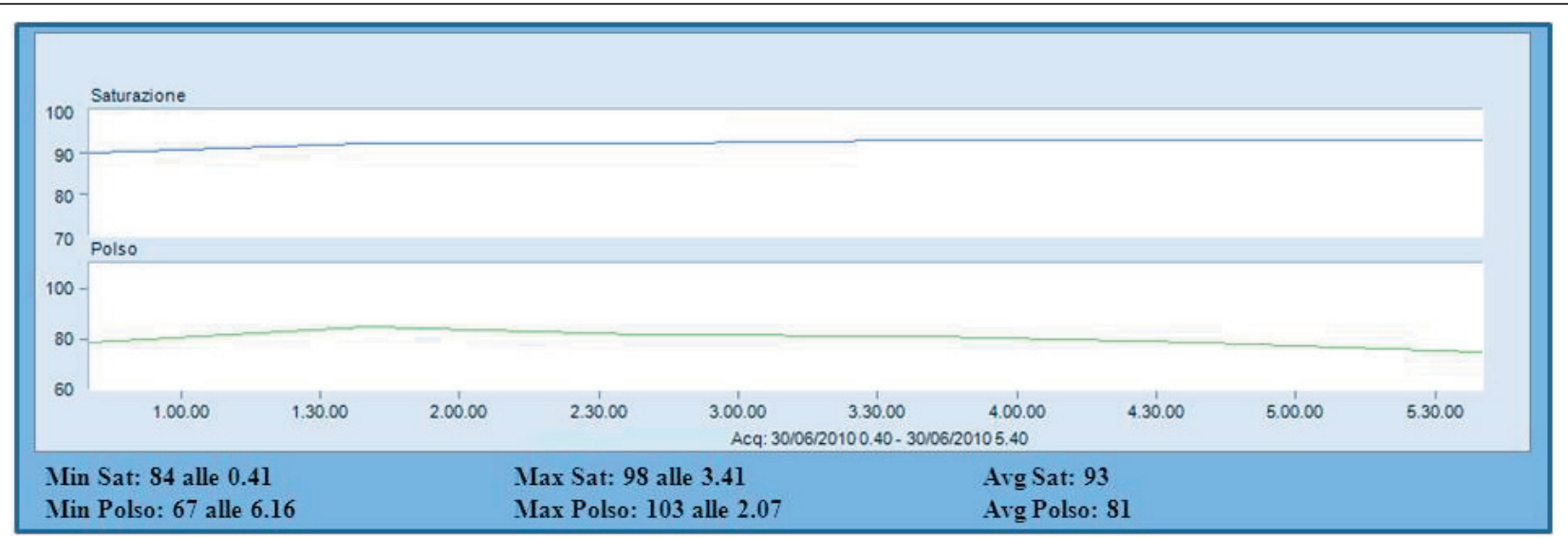

Fig. 3 - Examples of the recorded traces with the Sally System: Arterial Oxygen Saturation (SaO2) and Heart rate.

\section{Discussion}

The feasibility of a telemonitoring system may be inferred from the degree of patient's acceptance, easiness of use and economic sustainability. The number of times that the patient is connected, the total number of hours of connection, the average duration of each connection and the number of valid traces that the patient is able to send for each connection may be considered, together with costs, key points to establish the degree of feasibility. Based on the results obtained, in terms of numbers of times and of hours of connection, of duration of each connection and of number of traces sent for each con- nection especially in patients with good compliance, this pilot study showed that the telemonitoring system employed was sufficiently easy to use for the patient, and this was more evident in the group of patients that showed a good compliance to the study protocol (which included both the use of system for remote monitoring and periodic visits).

The level of satisfaction expressed by the patients for this model of telemonitoring was high (satisfaction index: $67 \%$ in all patients, that increased up to $89 \%$ in compliant patients). The perception of patients in terms of usefulness of the service, safety and facilitation in the management of the disease was mainly due to the possibility to have

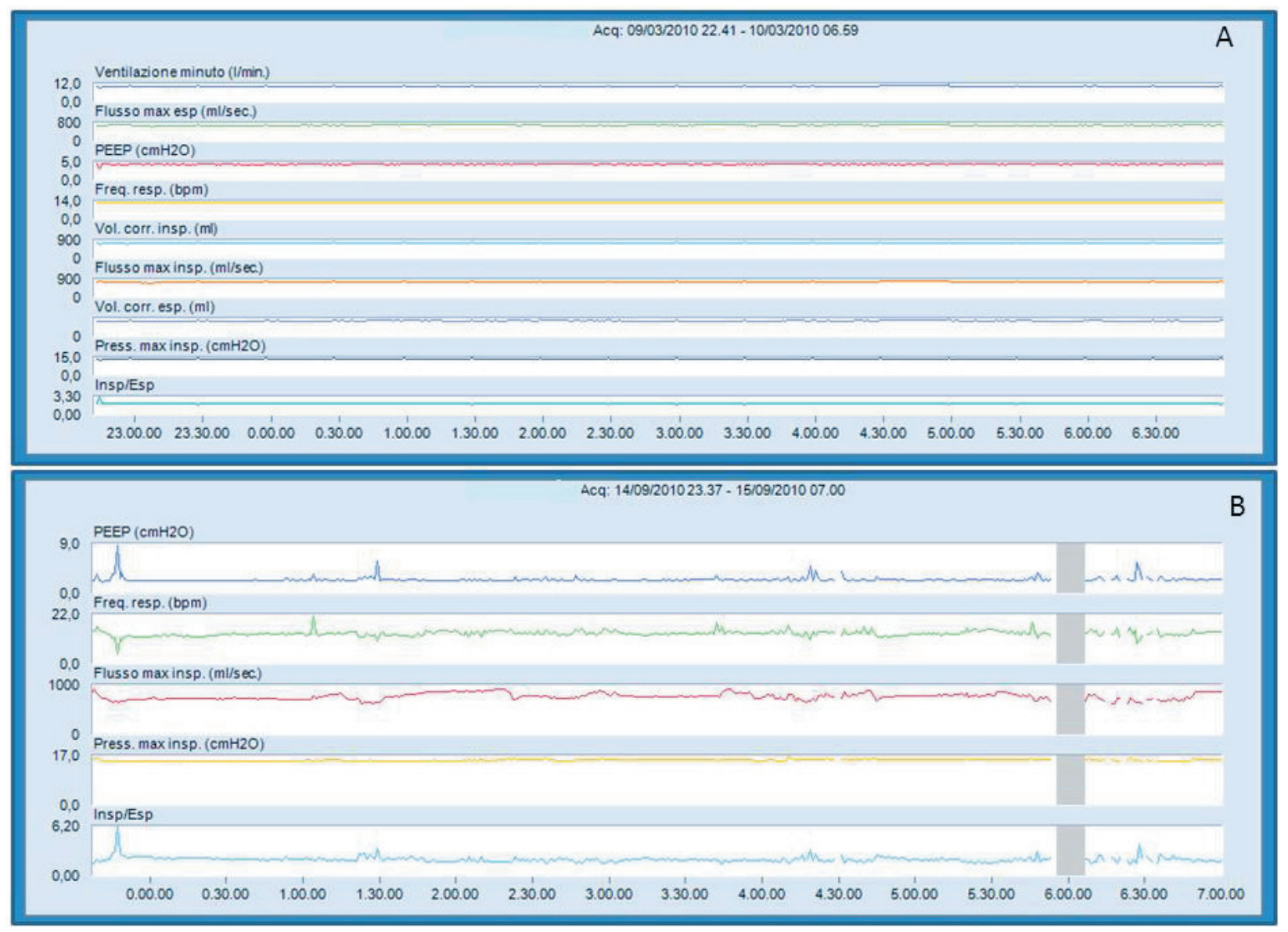

Fig. 4. - Examples of the recorded traces with the Sally System: in panel A traces of patient in Invasive Mechanical Ventilation, in panel B traces of patient in Noninvasive Mechanical Ventilation. 
Table 5. - Number of hospital admission and hospital stay

PANEL A: ALL PATIENTS (16)

\begin{tabular}{lccccccc}
\hline \multicolumn{7}{c}{ Two previous years } & At the end of the study \\
& Mean & SD & Mean & SD & 95\% CI for difference & P* \\
\hline Hospital admission, No & 0.8125 & 0.98 & 0.4375 & 1.09 & $-0.171-0.921$ & 0.164 \\
\hline Hospital Stay, days & 9.8 & 16.1 & 4.4 & 7.9 & $-3.34-14.2$ & 0.206 \\
\hline
\end{tabular}

PANEL B: PATIENTS WITH GOOD ADHERENCE TO THE PROTOCOL (9)

\begin{tabular}{lcccccc}
\hline & Mean & SD & Mean & SD & 95\% CI for difference & P* \\
\hline Hospital admission, No & 1.2 & 1.09 & 0.3 & 0.5 & $0.176-1.6$ & 0.021 \\
\hline Hospital Stay, days & 16 & 19.4 & 2.6 & 4.2 & $0.142-26.5$ & 0.048 \\
\hline *t-student for paired data. & & & & & &
\end{tabular}

a constant control of their clinical condition under a strict supervision, 24 hours/24 for 7 days a week, of a medical doctor operating in our Respiratory Intensive Care Unit in case of urgent need. Our telemonitoring system was able to reduce of $62,5 \%$ the emergency visits through direct communication between doctor and patient. Also the possibility to evaluate the recorded traces every week and the scheduled visits every two months increased the level of confidence of patients in this model.

The data of our study is in keeping with those previously published in the literature $[10,18,19$, 24] regarding a better control of patients with chronic respiratory failure obtained by monitoring cardio respiratory parameters at home in frail patients. Even though the results of these studies are quite similar the methods employed and the systems used were different.

In two studies $[10,20]$ the key role of nurses as specialised figures able to resolve alone clinical or logistical problems was reported. The model described by Vitacca [10] was able to reduce the number of hospitalisations of $36 \%$ in the study group, the number of urgent GP calls of $65 \%$ and the number of acute exacerbations of $71 \%$. Overall this system was able to reduce the costs of treatment without affecting the quality of assistance.

Pinto [18] in patients afflicted with ALS in noninvasive mechanical ventilation evaluated the role of telemonitoring at home. The patients were instructed to activate the system once a week or whenever they felt uncomfortable with the ventilator device after phoning the physician in charge at the hospital unit where the study was conducted. The doctor was able to modify the ventilator setting if needed or to schedule an office visit, schedule a phone call or conduct in real time communication. With this system the Authors showed in the study group in comparison to the control group a reduction of $66 \%$ of office visits, $80 \%$ of emergency room visits and $84 \%$ of hospital admissions.

Maiolo et al [19] reported that a telemedicine program based on determinations of arterial oxy- gen saturation and heart rate performed twice a week, coupled with periodic phone calls every week, was able to reduce hospital admissions by $50 \%$ and acute home exacerbations by $55 \%$.

None of the above mentioned studies reported the degree of compliance to the study protocol with the system employed. The level of satisfaction of the patients [19] and patients/caregivers [20] was reported in few studies.

As reported by Zanaboni [25] many factors are associated with successful telemedicine application including demonstrable savings, adequate financing, acceptance by clinicians, improved access to healthcare, avoidance of travel for patients in rural and remote areas and sustainability of the system. In our opinion another important factor is the acceptance of the patients and care givers of the systems and telemonitoring programme which is a critical point for a good compliance to the treatment. In several studies have been reported positive results in relation to acceptance of the systems and telemonitoring program [26]. However these studies dealt mainly with asthmatic patients and patients undergoing pulmonary transplantation.

Recently Zamith M et al [24], classifying the use of the system employed in their model of teleassistance as correct, partially correct and incorrect in 31 patients with Chronic Respiratory Insufficiency, reported a reduced number and duration of hospital admission with improvement in quality of life in the group of patients who used the system correctly.

In our study the model of telemonitoring employed was based on a regular transmission of data once a week and when needed via internet to the station located in RICU. The data was analysed routinely every week or in real time after a phone call by the patient or care givers to the doctor in charge. The telephone assistance and the evaluation of the signals on demand were available 24 hours $/ 24$ for 7 days/7. Our system was able to reduce the emergency visits in $62.5 \%$ of cases. Furthermore comparing the number of hospital admission and the days spent in hospital during the peri- 
od of study with the two previous years, we have found a trend in reduction of both variables in all patients studied. This data became statistically significant when we look at the patients with good adherence to the study protocol. Also the level of satisfaction was better in the more compliant patients.

The limits of the present study are: 1) the small number of the patients mainly due to the restriction criteria adopted, only patients resident in the province of Florence for technical reasons were considered eligible for the inclusion in the study; 2 ) the lack of cost analysis and cost benefit at the light of system employed; 3 ) the use of a not validated questionnaire for the evaluation of the level of satisfaction. Considering all these limitations, our results must be regarded with caution. However this must be considered as a pilot study lasting 2 years in a selected type of patients with a very critical chronic illness. Does this increases the problem of the extensive use of telemedicine for all patients or for a selected group which need a close surveillance? In our opinion is this last group of patients who may benefit better by the telemonitoring services. The home close monitoring of these frail patients may allow the detection of a clinical early deterioration that may be treated at home, avoiding hospitalisation which in turn generates high costs and a deterioration of the quality of life of patients and care givers. However, a recent systemic review of the studies of the cost-effectiveness of telemedicine and telecare concluded that there are no conclusive evidence that telemedicine and telecare intervention are cost-effective compared to conventional health care [27].

In conclusion: our pilot study showed that the telemonitoring system employed for patient with severe chronic ventilator failure in HMV was feasible and easy to use, and this was more evident in the group of patients that showed a good compliance to the study protocol; furthermore the perception of patients in terms of usefulness of the service, safety and facilitation in the management of the disease was higher in the same subgroup of patients.

\section{Appendix A}

\section{SATISFACTION SCORE BY TELEPHONE QUESTIONNAIRE}

a) Is the service of teleassistance useful?

$$
\begin{aligned}
& 0=\text { no/indifferent } \\
& 1=\text { yes, a little } \\
& 2=\text { yes, moderately } \\
& 3=\text { yes, completely }
\end{aligned}
$$

b) Do you feel save with this service?

$$
\begin{aligned}
& 0=\text { no/indifferent } \\
& 1=\text { yes, a little } \\
& 2=\text { yes, moderately } \\
& 3=\text { yes, completely }
\end{aligned}
$$

c) Has this service improved the management of your disease?

$$
\begin{aligned}
& 0=\text { no/indifferent } \\
& 1=\text { yes, a little } \\
& 2=\text { yes, moderately } \\
& 3=\text { yes, completely }
\end{aligned}
$$

Acknowledgements: The project of teleassistance for patients with chronic respiratory failure in home mechanical ventilation is contained in the Health Integrated Plan (PIS) 2005-2007 adopted by "Giunta della Salute di Firenze" (Resolution $\mathrm{n}^{\circ} 15$, on the 4 th November 2005) and approved by the City Council of Florence on the 5th December 2005). This study was supported by the tele@care service of ASP Montedomini, Firenze thanks to Dr.ssa L. Abamonti and Dr. A. Angeli.

\section{References}

1. Strong K, Mathers C, Leeder S, Beaglehole R. Preventing chronic diseases: how many lives can we save? Lancet 2005; 366: 1578-1582.

2. Lloyd-Owen SJ, Donaldson GC, Ambrosino N, et al. Patterns of home mechanical ventilation use in Europe: results from the Eurovent survey. Eur Respir J 2005; 25: 1025-1031.

3. Tuggey JM, Plant PK, Elliott MW. Domiciliary non-invasive ventilation for recurrent acidotic exacerbations of COPD: an economic analysis. Thorax 2003; 58: 867-71.

4. Chapman KR, Mannino DM, Soriano JB, et al. Epidemiology and costs of chronic obstructive pulmonary disease Eur Respir J 2006 27: 188-207.

5. Koleva D, Motterlini N, Banfi P, Garattini L "Healthcare costs of COPD in Italian referral centres: A prospective study". Respir Med 2007; 101: 2312-2320.

6. Young M, Sparrow D, Gottlieb D, Selim A, Friedman R. A telephone-linked computer system for COPD care. Chest 2001; 119: 1565-1575.

7. Agha Z, Schapira RM, Maker AH. Cost effectiveness of telemedicine for the delivery of outpatient pulmonary care to a rural population. Telemed J E Health 2002; 8: 281-291.

8. Vitacca M, Pizzocaro P, Assoni G, et al. A pilot study of nurse-led, home-based TP for 45 patients with chronic respiratory insufficiency with mechanical ventilation assistance. J Telemed Telecare 2006; 12: 337-342.

9. Giordano A, Scalvini S, Zanelli E, et al. Multicenter randomised trial on home based telemanagement to prevent hospital readmission of patients with chronic heart failure. Int J Cardiol 2009; 131: 192-9.

10. Vitacca M, Bianchi L, Guerra A, et al. Tele-assistance in chronic respiratory failure: a randomised clinical trial. Eur Respir J 2009; 33: 411-418.

11. American Thoracic Society. Statement on home care for patients with respiratory disorders. Am J Respir Crit Care Med 2005; 171: 1443-1464.

12. Farre R, Lloyd-Owen SJ, Ambrosino N, et al. Quality control of equipment in home mechanical ventilation: a European survey. Eur Respir J 2005; 26: 86-94.

13. Govoni L, Farré R, Pedotti A, Montserrat JM, Dellacà RL. An improved telemedicine system for remote titratio and optimization of home mechanical ventilation. Biomedical Engineering Conference (CIBEC), $20105^{\text {th }}$ Cairo International; 66-69.

14. Polisena J, Tran K, Cimon K. Home telehealth for chronic obstructive pulmonary disease: a systematic review and meta-analysis. J Telemed Telecare 2010; 16: 120-7.

15. Telehealthcare for chronic obstructive pulmonary disease. Cochrane Database Syst Rev 2011 Jul 6; (7): CD007718.

16. Bolton CE, Waters CS, Peirce S, Elwyn G; EPSRC and MRC Grand Challenge Team. Insufficient evidence of benefit: a systematic review of home telemonitoring for COPD. J Eval Clin Pract 2011 Dec; 17: 1216-22.

17. Lujan M, Moreno A, Veigas C, Montòn C, Pomares X, Domingo $\mathrm{C}$. Non-invasive home mechanical ventilation: effectiveness and efficiency of an outpatient initiation protocol compared with the standard in-hospital model. Respir Med 2007; 101: 1177-1182. 
18. Pinto A, Almeida JP, Pinto S, Pereira J, Oliveira AG, de Carvalho M. Home telemonitoring of noninvasive ventilation decreases healthcare utilisation in a prospective controlled trial of patients with amyotrophic lateral sclerosis. J Neurol Neurosurg Psychiatry 2010; 81: 1238-1242.

19. Maiolo C, Mohamed EI, Fiorani CM, De Lorenzo A. Home telemonitoring for patients with severe respiratory illnesses: the Italian experience. $J$ Telemed Telecare 2003; 9: 67-71.

20. Vitacca M, Comini L, Tentorio M, et al. A pilot trial of telemedicine-assisted, integrated care for patients with advanced amyotrophic lateral sclerosis and their caregivers. J Telemed Telecare 2010; 16: 83-88.

21. Tura A, Santini P, Longo D, Quareni L. A telemedicine instrument for home monitoring of patients with chronic respiratory diseases. Ann Ist Super Sanità 2007; 43: 101-109.
22. Fletcher C, Elmes P, Wood C. The significance of respiratory symptoms and the diagnosis of chronic bronchitis in a working population. BMJ 1959; 1: 257-26.

23. Miller MR, Hankinson J, Brusasco V, et al. Standardisation of Spirometry. Eur Respir J 2005; 26: 319-38.

24. Zamith M, Cardoso T, Matias I, Marques Gomez MJ. Home telemonitoring of severe chronic respiratory insufficient and asthmatic patients. Rev Port Pneumol 2009; 15: 385-417.

25. Zanaboni P, Wootton R. Adoption of telemedicine: from pilot stage to routine delivery. BMC Med Inform Decis Mak 2012 Jan 4; 12: 1.

26. Jaana M, Paré G, Sicotte C. Home telemonitoring for respiratory conditions: a systematic review. Am J Manag Care 2009; 15: 313-320.

27. Mistry H. Systematic review of studies of the cost-effectiveness of telemedicine and telecare. Changes in the economic evidence over twenty years. $J$ Telemed Telecare 2012; 18: 1-6.

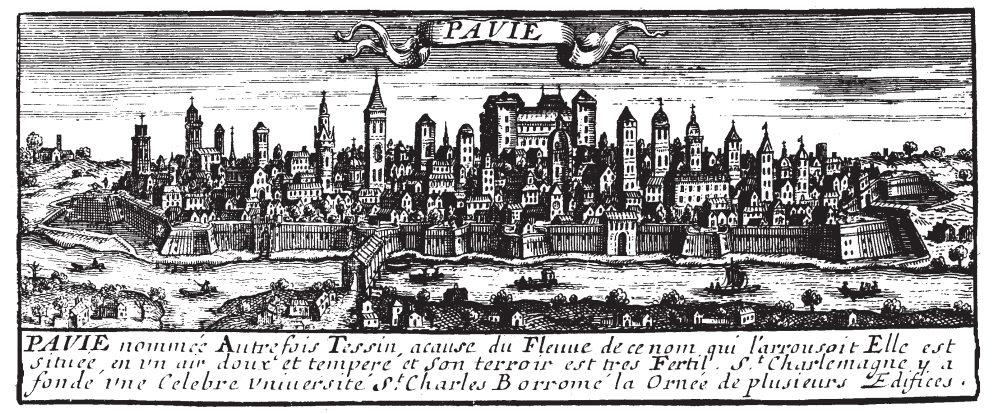

